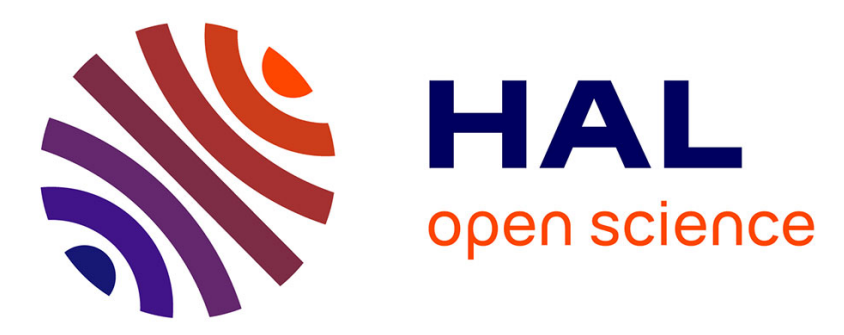

\title{
Optimizing the Multivalent Binding of the Bacterial Lectin LecA by Glycopeptide Dendrimers for Therapeutic Purposes
}

Benjamin Bouvier

\section{- To cite this version:}

Benjamin Bouvier. Optimizing the Multivalent Binding of the Bacterial Lectin LecA by Glycopeptide Dendrimers for Therapeutic Purposes. Journal of Chemical Information and Modeling, 2016, 56 (6), pp.1193-1204. 10.1021/acs.jcim.6b00146 . hal-02395197

\section{HAL Id: hal-02395197 \\ https://hal.science/hal-02395197}

Submitted on 9 Dec 2019

HAL is a multi-disciplinary open access archive for the deposit and dissemination of scientific research documents, whether they are published or not. The documents may come from teaching and research institutions in France or abroad, or from public or private research centers.
L'archive ouverte pluridisciplinaire HAL, est destinée au dépôt et à la diffusion de documents scientifiques de niveau recherche, publiés ou non, émanant des établissements d'enseignement et de recherche français ou étrangers, des laboratoires publics ou privés. 


\title{
Optimizing the Multivalent Binding of
}

\section{Bacterial Lectin LecA by Glycopeptide Dendrimers for Therapeutic Purposes}

\author{
Benjamin Bouvier* \\ Laboratoire de Glycochimie, des Antimicrobiens et des Agroressources, CNRS \\ UMR7378/Université de Picardie Jules Verne, 10, rue Baudelocque, 80039 Amiens Cedex, \\ France. \\ E-mail: benjamin.bouvier@u-picardie.fr
}

\begin{abstract}
Bacterial lectins are nonenzymatic, sugar-binding proteins involved in the formation of biofilms and the onset of virulence. The weakness of individual sugar-lectin interactions is compensated by the potentially large number of simultaneous copies of such contacts, resulting in high overall sugar-lectin affinities and marked specificities. Therapeutic compounds functionalized with sugar residues can only compete with host glycans for the binding to lectins if they are able to take profit of this multivalent binding mechanism. Glycopeptide dendrimers, featuring tree-like topologies with sugar moieties at their leaves, have already shown great promise in this regard. However, optimizing the dendrimers' aminoacid sequence is necessary to match the dynamics of the lectin active sites with that of the multivalent ligands. This work combines long-timescale, coarse-grained simulations of dendrimers and lectins with a reasoned exploration of the
\end{abstract}

*To whom correspondence should be addressed 
dendrimer sequence space, in an attempt to suggest sequences that could maximize multivalent binding to the galactose-specific bacterial lectin LecA. These candidates are validated by simulations of mixed dendrimer/lectin solutions, and the effects of the dendrimers on lectin dynamics are discussed. This approach is an attractive first step in the conception of therapeutic compounds based on the dendrimer scaffold, and contributes to the understanding of the various classes of multivalency which underpin the ubiquitous 'sugar code'.

\section{Introduction}

Carbohydrates play a prominent role in the mediation of biological processes, notably via the recognition of lectins by cellular glycans. This recognition process, dubbed the 'sugar code', ${ }^{1}$ remains the subject of active investigation, with fields of relevance ranging from oncology, ${ }^{2}$ through biosensors ${ }^{3}$ and synthetic supramolecular chemistry, ${ }^{4}$ to bionergy production ${ }^{5}$ and infectiology. ${ }^{6,7}$ Lectins feature a wide array of binding site topologies that can bind sugar moieties, albeit with affinities that are generally weak. Despite this, a very strong specificity

for certain cellular glycans can be achieved. ${ }^{1}$ This apparent contradiction can be resolved by introducing the concept of multivalency, in which multiple simultaneous weak interactions between carbohydrates and their receptors contribute to an overall strong affinity that can be fine-tuned through its individual components. ${ }^{8,9}$ Consequently, the conception of synthetic scaffolds bearing multiple covalently-bound carbohydrates as a means of taking advantage of the multivalency effect in the recognition and binding of lectins has been an ongoing research goal over the past decade. ${ }^{10-15}$

Dendrimers are molecules which feature a tree-like topology, with branches splitting off a trunk and then subdividing again following a regular pattern. The structural and dynamical properties of the branch termini, where functionalization usually takes place, depend on the chemical nature of the branches and the interactions between them, making dendrimers particulary versatile scaffolds in numerous molecular recognition applications - 
among which lectin/glycan recognition for which they have been used for several years. ${ }^{14,15}$ Peptidic dendrimers, inspired by the antimicrobial peptides which are a part of the defense mechanisms of most multicellular organisms, ${ }^{16}$ have the advantage of being very convenient to synthetize; as an added bonus, their compact yet flexible molten-globule structure makes them very resilient to proteolysis, ${ }^{17}$ facilitating their delivery. As potential antibiotic agents, they have already shown promising effects against the the human opportunistic bacterium Pseudomonas aeruginosa: nonfunctionalized, amphiphilic dendrimers have proven able to disrupt the bacterial membrane, ${ }^{18,19}$ whereas glycoconjugated peptidic dendrimers targeting bacterial lectins have been shown to hinder bacterial adhesion to human cells and biofilm formation, limiting the onset of virulence and resistance to antibiotics. ${ }^{15,20,21}$

Bacterial lectins such as the galactose-specific LecA and the fucose-specific LecB play a role in the specific recognition of host cells, the attachment to target cells, and the triggering of virulence. ${ }^{22,23}$ As tetramers, they feature four sugar-binding sites. Designing peptidic dendrimer glycoconjugates in which the spacing between sugar moieties coincides with the intra- and/or inter-tetramer binding site distances would, in theory, allow to maximize the impact of multivalency on the recognition and binding process and ensure optimal competition with the binding of host glycans, enhancing the therapeutic efficiency of the compounds. A thorough and systematic optimization of the aminoacid sequence of the dendrimer 'arms' to achieve this goal is therefore highly desirable, but also very difficult due to the unfavorable sequence combinatorics involved. In silico studies, which are in principle well suited to the cost-effective preliminary screening of a large number of candidates, also stumble on this difficulty: flexible molecular constructs such as peptide dendrimers have extensive conformational spaces that are very difficult to fully characterize at an acceptable computational cost. As such, theoretical studies on the matter have mostly been used either as a posteriori validation of experimental results, ${ }^{20}$ or to explore the effects of limited variations upon sequences gleaned from databases. ${ }^{24-26}$

Coarse-grained models (in which atoms making up standalone chemical functions are 
grouped into beads) can be used to simulate much longer timescales than their all-atom counterparts for the same computational cost; this is not only due to the straightforward reduction in the dimensionality of the problem, but also to an effective kinetic speedup associated with the 'smoothing out' of local features on the energy landscape brought about by coarse-graining. ${ }^{27}$ This study applies the MARTINI ${ }^{28,29}$ coarse-grained model (which has been successfully applied to unstructured antimicrobial peptides in the past ${ }^{30-32}$ ) and long-timescale molecular dynamics simulations to the systematic exploration of aminoacid sequence effects on the dynamics of octavalent peptide dendrimers functionalized with galactose, as well as their ability to bind the galactose-specific lectin LecA. The sequence-todynamics relationship thus inferred is then used to suggest potential candidates in which the recognition and binding of LecA would benefit the most from multivalency. Finally, the best and worst candidates are simulated in the presence of LecA tetramers to evaluate the validity of these predictions.

\section{Methods}

\section{Root mean-square deviation calculations}

Due to the topology of the peptide dendrimers considered (see Figure 1), aminoacid chains branching from the same parent residue are chemically equivalent, and their numbering in the structure can be freely swapped. This has to be taken into account when computing the root mean-square deviation ${ }^{33}$ (RMSD) between two dendrimer structures: both the preliminary least-square alignment procedure (used to remove the effects of rigid-body rotation and translation) and actual deviation calculation require a mapping of atoms between both structures, which for such dendrimers is not unique. The ability to swap equivalent branches results in two possible numberings per branching point. The dendrimers studied herein have seven branching points, for a total of $2^{7}=128$ possible equivalent numberings. The RMSD between two structures can thus be computed as the minimum of the set of RMSDs ob- 
tained between structure 1 and the 128 equivalent numberings of structure 2. A tool for the automatic generation of all possible mappings between structures and calculation of the corresponding RMSDs has been designed for this study.

\section{Statistical tools}

The Kolmogorov-Smirnov (KS) distance ${ }^{34}$ was used to quantify the similarity between distributions of structural values (RMSD, inter-sugar distances...) obtained from the simulation of the different dendrimer compounds. The clustering of dendrimers was performed using the affinity propagation method. ${ }^{35}$ The method iteratively computes the pairwise responsibility $r(i, j)$ and availability $a(i, j)$ between pairs of samples $(i, j)$ taken from a dataset. Responsibility is the accumulated evidence that sample $j$ should be an exemplar for sample $i$ (in other words, that sample $j$ should be the representative structure of a cluster containing both itself and sample $i$ ); availability is the accumulated evidence that sample $i$ should choose sample $j$ as its exemplar, when compared to sample $j$ 's exemplarity for all other samples:

$$
\left\{\begin{array}{l}
r(i, j)=s(i, j)-\max [a(i, k)+s(i, k) \forall k \neq j] \\
a(i, j)=\min \left[0, r(j, j)+\sum_{k, k \notin\{i, j\}} r(k, j)\right]
\end{array}\right.
$$

$s(i, j)$ is the similarity between samples $i$ and $j$, which was taken as the opposite value of the KS distance between the corresponding distributions. The system of equations (1) is iterated to convergence. The affinity propagation method was chosen because of its ability to take the similarities between samples, rather than the samples themselves, as input; this is very convenient when clustering complex objects (such as distributions) using nonstandard metrics. As an additional benefit, affinity propagation does not require the number of clusters to be specified by the user, a source of bias found in most other clustering techniques. However, on complex datasets, the method has a tendency to generate many clusters. To alleviate this behavior, affinity propagation was performed hierarchically on the datasets: 
the exemplars generated at generation $n$ were used as the input set for generation $n-1$; in this scheme, the generation $n$-exemplars are children of the generation $n$ - 1-exemplars to whose cluster they belong. The process was repeated until further clustering did not result in a simplification of the dataset. This hierarchical approach retains the advantage of not having to choose the number of clusters, yet provides different levels of classification that facilitate the understanding of the structure of the underlying dataset by the human mind.

Sequence logos are a widely used representation of multiple sequence alignments of proteins or nucleic acids. ${ }^{36}$ A logo consists of a stack of symbols per position in the sequence; the height of each character in the stack relates to the frequency of the corresponding aminoacid/nucleobase at the position, while the total height of the stack denotes sequence conservation. The latter is expressed as the difference between the maximum Shannon entropy $S_{\max }$ and the entropy in the observed character distribution at the considered position $S_{o b s}:$

$$
R_{s e q}=S_{m a x}-S_{o b s}=\log _{2} N-\left(-\sum_{n=1}^{N} p_{n} \log _{2} p_{n}\right)
$$

where $p_{n}$ is the frequency of symbol $n$ at the location, and $N$ is the number of possible distinct symbols.

\section{Computational details}

All coarse-grained MD simulations were performed using version 2.2 of the MARTINI force field $^{28,29}$ inside GROMACS ${ }^{37,38}$ versions 4.6 .5 and 5.1. The parameters for the branching lysines were derived from those of the standard aminoacid, ${ }^{28}$ replacing the final Qd-type

grain by a P1-type particle which was bound to the backbone grain of the next aminoacid (bond equilibrium length $0.35 \mathrm{~nm}$, bond force constant $1250 \mathrm{~kJ} \mathrm{~mol}^{-1} \mathrm{~nm}^{-2}$; angle equilibrium value $124^{\circ}$, angle force constant $20 \mathrm{~kJ} \mathrm{~mol}^{-1} \mathrm{rad}^{-2}$ ). These parameters were chosen to provide the best match with the corresponding all-atom model on a set of distance distributions (see 
Supporting Information Figure S1 for details). The parameters for the terminal galactose residues were taken from Lopez and coworkers' extension of MARTINI to carbohydrates. ${ }^{39}$ The sugars were covalently bound to the backbone bead of the corresponding N-terminal residue (bond equilibrium length $0.35 \mathrm{~nm}$, bond force constant $1250 \mathrm{~kJ} \mathrm{~mol}^{-1} \mathrm{~nm}^{-2}$; angle to sugar bead 1: equilibrium value $127^{\circ}$, force constant $20 \mathrm{~kJ} \mathrm{~mol}^{-1} \mathrm{rad}^{-2}$; angle to sugar bead 2: equilibrium value $149^{\circ}$, force constant: $25 \mathrm{~kJ} \mathrm{~mol}^{-1} \mathrm{rad}^{-2}$; angle to sugar bead 3 : equilibrium value $123^{\circ}$, force constant: $25 \mathrm{~kJ} \mathrm{~mol}^{-1} \mathrm{rad}^{-2}$ ). The dendrimers were treated as extended regions (coils) within the MARTINI framework, and as such did not feature secondary structure restraining potentials.

The dendrimers were minimized in vacuo, placed in a truncated octahedral box of MARTINI water beads (in which one bead replaces 4 actual water molecules ${ }^{28}$ ) extending at least $14 \AA$ from the solute in all directions. Interestingly, while the simple MARTINI water model cannot account for the possible mediation of contacts by single water molecules or the directionality of hydrogen bonding patterns, the bundling of water molecules inside a bead has been shown to have little overall effect on the physical and thermodynamical aspects of the interaction of water with most solutes. ${ }^{40}$ The solvated system was then minimized to convergence and equilibrated at $300 \mathrm{~K}$ with fixed backbone beads for $5 \mathrm{~ns}$. Production simulations without restraints were then performed for $5 \mu$ s. All molecular dynamics simulations used a timestep of $20 \mathrm{fs}$. Conditions of constant temperature (300 K) and pressure (1 bar) were maintained using velocity-rescaling $(\tau=1.0 \mathrm{ps})$ and Parrinello-Rahman algorithms $\left(\tau=12 \mathrm{ps}, \beta=1.8 \times 10^{-5} \mathrm{bar}^{-1}\right)$, respectively, applied separately to solute and solvent. Coulomb interactions extended to $12 \AA$, with the forces decaying smoothly from $0 \AA$. Van der Waals interactions extended to $12 \AA$, with the forces decaying smoothly from $9 \AA$.

The structure of the LecA tetramer was taken from the work of Novoa and coworkers ${ }^{20}$ (PDB Id. 4CP9), converted to a coarse-grain representation using Martinize, and minimized in vacuo. As is usual for globular proteins in MARTINI, the secondary structure of each of the monomers was preserved by employing an additional elastic network model connecting 
the backbone beads of aminoacids distant of less than $9 \AA$ and separated by at least 3 sequence positions (force constant $500 \mathrm{~kJ} \mathrm{~mol}^{-1} \mathrm{~nm}^{-2}$ ). Spurious LecA tetramer dissociation events, although rarely observed, could have a negative impact on the efficiency of conformational sampling; to prevent them, the disposition of the LecA units inside a tetramer was constrained using harmonic potentials between the centers of masses of the units $(500 \mathrm{~kJ}$ $\left.\mathrm{mol}^{-1} \mathrm{~nm}^{-2}\right)$. Similarly, rare exchanges of the $\mathrm{Ca}^{2+}$ ion at each monomer's binding site were seen to occur, over timescales compatible with the ion's experimental binding affinity; ${ }^{41}$ to prevent such 'blue moon' events from tainting the sampling of galactose recognition and binding events, the ions were restrained relative to the backbone beads of active site aminoacids Tyr36 and Thr104 with a $500 \mathrm{~kJ} \mathrm{~mol}^{-1} \mathrm{~nm}^{-2}$ harmonic potential. Five copies of the minimized LecA tetramer were inserted, at random positions and orientations, inside an octahedral box which was then filled with MARTINI water beads. Minimization and equilibration were performed as previously described, upon which $2 \mu$ s of unrestrained production simulation were begun. This was repeated for three distinct starting conditions (different random arrangements of the five tetramers), yielding $6 \mu$ s of total simulation time. In all cases, the equilibrated box had a typical volume of around $6000 \mathrm{~nm}^{3}$, corresponding to an approximate LecA concentration of $1.4 \mathrm{mM}$, and comprised approximately 48000 beads.

The simulation of the LecA/dendrimer mixtures were carried out similarly, by randomly introducing five copies of the relevant dendrimer and five LecA tetramers into the simulation cell. Three distinct starting conditions were thus generated, in which (to ensure generality) the absence of initial dendrimer-lectin contacts was checked. The protocols and simulation lenghts were the same as for the $\mathrm{LecA}_{5}$ system; the approximate volume of the equilibrated box, the number of particles, and the concentrations of LecA and dendrimers were also very similar to their values for $\mathrm{LecA}_{5}$. For each dendrimer, an additional set of three 2us simulations from distinct starting conditions was generated to verify the convergence of active site distance distributions (see Results for details). 


\section{Miscellaneous}

All statistical analyses were performed using Scientific Python ${ }^{42}$ and Scikit-learn. ${ }^{43}$ All molecular graphics were produced using VMD. ${ }^{44}$ Sequence logos were generated using the WebLogo Python API. ${ }^{45}$ Trees were represented using the ETE toolkit. ${ }^{46}$ All other plots and figures were generated using Matplotlib. ${ }^{47}$

\section{Results}

The functionalized peptide dendrimers under study possess a branching, tree-like topology with 8 'leaf' nodes, each of which bears a galactose moiety (see Figure 1). Suitably functionalized, branching peptide dendrimers of this nature have been shown to be highly effective in the recognition and binding of both the galactose-specific $\operatorname{Lec}^{21}$ and the fucose-specific LecB lectins. ${ }^{48}$ The particular framework selected for this study was chosen to be a good representative of this class: the octavalent scaffold can potentially maximize multivalent effects, and the use of a common-length spacer between any two branching points allows a consistent comparison of sequence effects as a function of residue depth. The framework consists of chains of homogeneous sequences: at any given depth in the tree, only one sort of aminoacid is found. There are 11 such levels, three of which correspond to branching lysine residues that cannot be replaced by another aminoacid because they effectively implement the tree topology. Consequently, 8 levels remain for the variation of the dendrimer aminoacid sequence.

The number of possible sequences, in the order of 25 billions $\left(20^{8}\right)$, precludes an exhaustive study even at the coarse-grained level. The number of aminoacid types employed to generate the dendrimer sequences has thus been reduced to five, representative of the major classes: small hydrophobic (alanine, A), large hydrophobic (methionine, M), polar neutral (serine, S), positively charged (arginine, R), negatively charged (aspartate, D). Proline (P), whose unique conformational rigidity makes it stand out from the group of hydrophobic aminoacids 
with which it is usually bundled, was verified to be reasonably represented by alanine for this study (see below and Supporting Information Figure S5). Although volume and chemical character are not independent variables, this subset of aminoacids has also been chosen as reasonably representative of the entire set of aminoacid volumes in solution ${ }^{49}(75.1 \pm 21.8$ vs $\left.85.1 \pm 24.0 \mathrm{~mL} \mathrm{~mol}^{-1}\right)$.

To further simplify the combinatorics of the problem, it is possible to vary the sequence inside a sliding window rather than on a per-residue basis; the number of possible sequences is thus reduced from $N^{L}$ to $(L-W+1) \times N^{W}$, where $N$ is the number of possible residue types per position, $L$ is the number of positions at which the sequence is varied and $W$ is the width of the sliding window. Longer windows are more costly, but offer a better description of the collective effects between neighboring residues. The dendrimers under study consist of branching lysines separating spans of two aminoacids; the lysine residues act as buffers between the dipeptides on either side, interfering with the propagation of correlated information between them. Consequently, using a window length of two in these systems was deemed a good tradeoff between computational cost and the inclusion of collective effects (a hypothesis that will be verified further down); it reduced from $\sim 391000$ to 175 the number of sequences to be considered, allowing for adequately long simulation times on each. The sequence variations were performed upon a dendrimer of sequence $\mathrm{H}_{2} \mathrm{~N}-\mathrm{IH}(\mathrm{K}) \mathrm{IK}(\mathrm{K}) \mathrm{FL}(\mathrm{K}) \mathrm{PK}$ Gal (from trunk to leaves, with branching lysines between parentheses), a variant of which has been experimentally shown to be a good binder of the lectin LecB (25 nM binding affinity); ${ }^{48}$ although the system-wide sequence exploration subsequently performed renders this choice somewhat irrelevant, it is justified by the similarities between LecA and LecB (charged active sites, comparable shortest intra-tetramer active site distances). The solubility of dendrimers in water, essential to their drugability, is theoretically favored by the presence of polar and charged aminoacids; however, the high number of galactose moieites per dendrimer and their location at the extremities of flexible chains, combined with the very high solubility of galactose $\left(\sim 4 \mathrm{~mol} \mathrm{~L}^{-1}\right)$, allow for a hydrophilic external shell that 
can potentially shield hydrophobic aminoacids from water, akin to the hydrophobic collapse observed in protein folding. As such, solubility issues at millimolar or lower concentrations are not expected regardless of sequence. Indeed, similar glycopeptide dendrimers have been shown to be water-soluble at such concentrations during isothermal titration calorimetry experiments. ${ }^{21,26}$

The 175 possible sequences have been constructed and simulated in explicit solvent for $5 \mu$ s. Quantifying differences between peptide structures is traditionally performed by computing the root mean-square deviation (RMSD) of the backbone atoms, after removal of rigid-body rotation and translation effects via least-square fitting; this approach has already been applied to peptidic dendrimers of varying sequences. ${ }^{25}$ The methodology was adapted to the symmetric topology of the current dendrimers (see Methods for details). The 'central' structure of each dendrimer (whose RMSD to the average structure over the entire simulation is minimal) was extracted, and a square, symmetric, rank-175 similarity matrix was built from the RMSD values between the central structures of all possible dendrimer pairs. This matrix was used as input to the affinity propagation clustering method (see Methods for details), which grouped the 175 dendrimers into six unevenly populated clusters. To visualize the properties of the clusters thus obtained, statistics of hydropathy indices ${ }^{50}$ (a well-adopted indication of an aminoacid's polarity), volumes and sequence positions were performed over each of them. The volume and hydropathy values for each dendrimer were taken as the sum of the corresponding values for the two aminoacids forming the mutation window; the sequence position was taken to be the index of the aminoacid within the window that is closest to the dendrimer trunk (Figure 2).

As can be seen, the clusters show no clear discrimination of dendrimers based on position, hydropathy or volume. All clusters contain dendrimers mutated on at least six of the seven possible positions with residues of all sizes and hydropathy; only a limited specificity for small and hydrophobic residues can be observed in cluster number 5 of population 18 . This is in part due to the fact that the RMSD is a very degenerate measure of structural differences: two 
structures with equal RMSD to a third are not necessarily similar, especially if the common RMSD is large; the RMSD is more sensitive to minute differences between structures than to very large ones. However, clustering based on this measure still makes a degree of sense since pairwise RMSDs inside each cluster are expected to be small if the number of clusters is sufficiently large (in the present case, the mean intracluster RMSD was found equal to $2.1 \AA$, which is indeed small compared to the mean intercluster RMSD of $6.4 \AA$ ). So one has to conclude that mutations in the dendrimer sequence have little impact on the mean structure. The other structural measure usually employed in such cases, the macromolecular radius of gyration, was also verified to feature a relative insensitivity to aminoacid nature (data not shown).

The average backbone structures of the dendrimer representative of each of the six clusters, aligned along their inertial axes, are presented on Figure 3. The figure also provides, for each cluster, the moment along each of the three inertial axes. As can be seen, the average structures of the dendrimers are quite diverse, ranging from extended (purple structure - RR mutation on the last branch) to compact (orange structure - AA mutation on the middle branch), and from highly isotropic (red structure - AS mutation on the last middle-branch and first last-branch residues) to highly anisotropic (purple structure). The existence of this structural diversity shows that the sequence of the dendrimer chains does have an impact on the systems' behavior, but that the static picture provided by the use of degenerate structural measures (RMSD, radii of gyration) on time-averaged structures is not suitable to its elucidation. There is a clear need to take into account the dynamics of the dendrimers in the classification mechanism (different dendrimer sequences could be characterized by different dynamics around similar average structures), and to optimize the dynamics-activity relationship using statistics over structural measures that relate as closely as possible to lectin recognition.

To this end, I have focused on the distribution of distances between sugar 'leaves' over time, as revealed by the $5 \mu$ s of MD simulation of each dendrimer sequence. This measure 
of the dendrimers' dynamical behavior does not suffer from the issues mentioned above, and is critically related to the ability of the dendrimers to multivalently bind LecA (see Supporting Information Figure S2 for examples of such distributions). A similarity matrix between all possible pairs of distributions was calculated based on the Kolmogorov-Smirnov distance (see Methods for details). As before, this matrix was subjected to clustering using affinity propagation; this yielded 28 distinct clusters - a testimony to the complexity of the underlying dataset. To facilitate the understanding of these results, a hierarchical approach was employed to generate larger 'parent' clusters. To visualize the nature of the clusters in the resulting 'tree', statistics of hydropathy indices, volumes and sequence positions were performed over each of them as previously described. Figure 4 represents the cluster tree, decorated with these per-cluster statistics.

Unlike before, there is now a clear influence of the position at which the sequence is varied on the constitution of the clusters; the impact of position on the overall dynamics of the systems appears much larger than that of the chemical nature or size of the aminoacids introduced. Three of the six first-generation clusters are exclusively composed of dendrimers mutated on the last branch; the fourth cluster mixes in a limited contribution of the second aminoacid of the middle branch. The two remaining clusters, much larger in population (28 and 124 members vs. a total of 23 for the previously mentioned four clusters), are mostly composed of dendrimers mutated on (respectively) the second and third, or the first and second, branches. The first-generation clusters composed of last-branch mutants show a limited, but apparent, discrimination between aminoacid polarities and sizes: the RR mutation of the last branch is alone in its cluster; the remainder of the hydrophilic combinations are divided into two clusters (size 3, small aminoacids, and size 6, larger ones); hydrophobic combinations of all sizes are bundled into the fourth cluster of population 13, for which discrimination over aminoacid sizes only occurs at generation 2. On the other hand, the two large first-generation clusters consisting of first to third-branch mutants do not give rise, at the second generation, to a marked separation based on size or hydropathy; 
the corresponding second-generation clusters are complex mixtures of dendrimer sequences, most of which are difficult to describe in simple terms. To summarize, the position of the mutation on the chains is the most important parameter to take into account when optimizing dendrimer sequences, followed by polarity, then by size; in practice, the last two properties only seem to matter for last-branch mutants. Interestingly, the dependence of structure and dynamics upon the polarity of the dendrimer arm aminoacids is strikingly more complex than the expected simple preference of polar (resp. hydrophobic) sequences for extended (resp. compact) conformations.

We now focus on collective effects on the dendrimer dynamics induced by the mutation of neighboring residues. Considering the fact that the aminoacid sequence was varied inside a sliding window of length 2 , there are 10 instances of dendrimers having a given mutation at a given position ( 5 possible neighbors on either side), except for the first and final positions which, having only one neighbor, are represented by 5 instances only. To evaluate the importance of neighbor effects for a given mutation at a given position, the average and standard deviation of the set of dissimilarities between all possible pairs of instances (the 'signal') were compared to the same statistical measures on the set of dissimilarities between each instance and all other sequences in the dataset (the 'background'). A signal that is lower than the background means that neighbor effects are not important; conversely, similar values of signal and background hint at sizeable neighbor effects. The left panel of Figure 5 shows that, for the dendrimers under study, three domains can be distinguished. From positions 1 to 7 , the average signal is clearly lower than the background and its spread is much reduced, meaning that it is probably safe to consider point mutations on an individual basis; on the other hand, at positions 8 and 10, neighbor effects seem important and varying the sequence over two or more positions at a time is mandatory; finally, neighbor effects abate again on average at position 11, although the spread in values remains high. The nature of the aminoacid, on the other hand, does not seem to consistently affect neighbor effects: aminoacid types for which neighbor mutations resulted in dissimilar sugar distance 
distributions were found to differ depending on their positions. Just like for the clustering discussed above, the position in the dendrimer sequence has the greatest impact on collective sequence effects. To check whether this effect is directional, the right panel of Figure 5 shows the mean and standard deviation of the dissimilarities of sugar distance distributions generated when changing the left (trunk-side) or right (leaf-side) neighbor of an aminoacid at a given position. The trunkward (left) part of the sequence, up to the end of the first branch (position 5), does not show significantly different right-side and left-side correlations. When moving toward the leaves however, the average KS distance between distributions for right-side mutations becomes markedly higher than for left-side ones.

While it may indeed seem natural that the dynamics of the sugar residues is most influenced by the close-lying (leafward) aminoacids, it is quite remarkable that the nature of 'parent' branches in the dendrimer topology seems to have little effect on the behavior of their 'children', even though the flow of correlation is expected to be directed from the dendrimer core to the branches.

The ability for a dendrimer of given sequence to multivalently bind LecA is very likely to depend on the extent of the overlap between its distribution of sugar distances and the distribution of LecA active site distances. To obtain the latter, five LecA tetramers were simulated at the coarse-grained level, in a solvent box corresponding to a concentration of $1.4 \mathrm{mM}$, for a total of $6 \mu \mathrm{s}$, and all possible distances between active site pairs were computed (a total of 190 per time step). As before, the overlaps between this distribution and each of the dendrimer sugar distance datasets were computed using the KS distance. The $20 \%$ of sequences with the best overlaps were used as input to the calculation of the per-position Shannon entropy; the resulting logo, appearing on Figure 6, shows the aminoacid preference at each position in the dendrimer sequence with respect to the multivalent binding of LecA (insofar as the hypothesis that the overlap of distance distributions between receptors and ligands correlates with multivalency is correct).

The sequence logo comforts the previous finding that the last branch aminoacids play a 
crucial role in dendrimer dynamics; there is an almost equally high specificity for serine or alanine at the corresponding positions. However, the sequence of the first branch also appears important, with a marked preference for aspartate at position 4 and its absence at position 5 (along with methionine at both). The dendrimer trunk features a marked preference for arginine at position 1 . The rest of the sequence is not as constrained, but displays an overall preference for either serine or alanine. To check whether the bundling of proline within the group of hydrophobic aminoacids despite its unique conformational rigidity is reasonable, proline was added to the previous set of 5 aminoacids for the generation of all possible sequences of the last dendrimer branch (in which sequence effects have just been shown to be the strongest). The resulting sequence logo, shown on Supporting Information Figure S5, shows that while the presence of a proline residue at the most leafward position is indeed favorable, it isn't more so than alanine: for the binding to LecA, the hydrophobic character of proline appears to prevail over its peculiar dynamical characteristics. As an aside, the fact that several experimentally validated dendrimers also feature leafward prolines ${ }^{21,48,51}$ provides additional evidence of the predictive power of the model employed in this work.

To check whether the sugar distance properties inferred from simulations on a set of dendrimers mutated inside a two-aminoacid sliding window (effectively neglecting correlations between second-neighbors and beyond) could be used to predict the entire dendrimer sequence, the sequences with the best and worst overlaps with the distribution of LecA active sites (as predicted from the sequence logo on Figure 6) were simulated under the same conditions as previously. The best sequence was RSKDAKASKSA. Due to multiple aminoacids with zero occurence at positions 5 and 11, several choices are possible for the worst sequence; ADKMMKMDKMM was chosen here. With a KS distance of 0.16 to the distribution of LecA binding site distances, the best sequence was indeed found to be better than any other of the 175 previously simulated sequences, while the worst sequence (with a KS distance of 0.32 ) was found to be worse than $98.9 \%$ of the sequence set (the histogram of $\mathrm{KS}$ distances to the LecA active site distance distribution for all dendrimer sequences 
under study can be found on Figure S3, Supporting Information). While this does not constitute proof that the best sequence is indeed the very best (which would require the entire set of $5^{8}$ sequences to be simulated), it provides convincing evidence that potential collective effects do not reach far enough along the sequence, or are not important enough, to nullify the previously made hypothesis of the additivity of two-aminoacid spans.

Finally, to check if the overlap of distance distributions between receptors and ligands can be considered a good measure of multivalency, and to evaluate the effect of the dendrimers on the dynamics of LecA tetramers, I have simulated the best and worst dendrimer sequences in the presence of LecA ( 5 LecA tetramers and 5 dendrimer copies, see Methods for details) - possibly the most realistic simulation of multivalent lectin binding to date, albeit tempered by the simplicity of the coarse-grained model used and the relatively high LecA concentration chosen to reduce computational costs. First, the effect of the dendrimers on the distribution of distances between active sites on different LecA tetramers was monitored (Figure 7); due to the rigidity of the LecA tetramer assembly, intra-tetramer active site distances are the same in all cases and were disregarded). The distribution of distances observed in the absence of dendrimers is not very strongly affected by the addition of the best dendrimer candidate: apart from the apparition of small peaks from 40 to $70 \AA$, the overall distribution remains mostly devoid of salient features (narrow, well-defined peaks). The distributions are characteristic of systems with high mobility, where multiple interactions occur on comparatively short timescales (Figure 8 shows the structure of a typical multivalent complex encountered during the simulation). On the other hand, the addition of the worst dendrimer candidate to a solution of LecA tetramers profoundly affects the distribution of active site distances, which now features well-defined peaks corresponding to long-lasting aggregates. The mobility of the LecA tetramers relative to one another has been reduced by the addition of the dendrimer, which brings about a structuration of the system. This is not incompatible with multivalency - actually, long-lasting LecA-tetramer aggregates could conceivably facilitate the simultaneous binding of dendrimers to multiple tetramers. Conversely, 
a high mobility and interactions over smaller timescales certainly promotes multivalency via facilitated diffusion. The satisfactory convergence of the distributions with respect to simulation lengths was checked by running, for both dendrimers, an additional $6 \mu$ s of simulation from independent starting conditions and comparing the resulting distributions to the ones discussed here (see Supporting Information Figure S4).

To examine in more detail the occurrence of multivalent dendrimer-lectin interactions in both cases, the number of lectin active sites within a certain distance of all dendrimer sugar moieties was computed over the trajectories and plotted on Figure 9. For the best dendrimer candidate, a rapid onset of potential multivalency can be seen: there is a nonzero (if small) probability that all five simulated LecA tetramers are within distances as small as 7.5 to $10 \AA$ of a galactose, and this probability increases linearly with distance. On the other hand, for the worst dendrimer a negligible probability of encountering four or five LecA tetramers is still observed even at distances as large as 22.5 to $25 \AA$; conversely, due to the greater ordering of LecA tetramers, the overall probability of encountering any number of LecA tetramers is consistently larger than for the best dendrimer candidate at distances larger than $5 \AA$.

\section{Discussion}

Simulating multivalent lectin recognition and binding in silico is a difficult task. Dendrimers are flexible molecules with large conformational spaces; encounters between dendrimers and lectins are diffusive in nature and as such, occur over long timescales; the relative weakness of dendrimer-lectin interactions makes it difficult to distinguish relevant binding modes from random encounter complexes (which could also be yet unidentified, alternate binding sites, contributing to the complexity of the sugar code which is, at the time being, still largely beyond our grasp ${ }^{1}$ ). As such, previous studies have often simulated dendrimers and lectins on their own, or focused on intra-tetramer (chelation) multivalency. Only very re- 
cently have all-atom simulations of inter-LecA multivalency been published, ${ }^{51}$ although the timescales involved (on the order of $10 \mathrm{~ns}$ ) preclude any major structural rearrangements or the possibility of observing multiple unbinding and rebinding events. By using a coarsegrain model which has been thoroughly validated both on protein and carbohydrates, this study trades the precise, physics-based description of atomic interactions against the possibility of reaching meaningful timescales on large systems containing both partners and their aqueous environment (due in part to accelerated kinetics), and considering sequence effects over a relatively large set of dendrimer sequences. Despite this, numerous limitations still remain. For instance, the concentration of lectins and dendrimers employed were deliberately overestimated compared to their typical experimental values, to limit the dimension of the simulation cells and facilitate encounters; this particularly applies to the dendrimers, some of which have shown lectin-binding activity at stoichiometries as low as 1:20000 in experiments! ${ }^{52}$ Other potential issues are linked to the MARTINI framework: the relative over-attractiveness of protein-protein interactions in MARTINI ${ }^{53}$ could overemphasize the statistical relevance of long-lived aggregates observed in, e.g., the 'worst' dendrimer candidate; the conformational space of unstructured peptides may not be as faithfully rendered in MARTINI as in an all-atom model, despite a growing corpus of successful applications of MARTINI to unstructured antimicrobial peptides. ${ }^{30-32}$ Considering that these issues should equally affect all dendrimer sequences, which can still be compared on a relative (if not absolute) basis, the tradeoff was deemed worthy of being adopted.

The first interesting point raised by this work is the non-trivial effect of the dendrimer aminoacid sequence on the dynamical properties of the dendrimers - whether isolated or in solution with LecA. In agreement with previous atomistic simulations, ${ }^{24}$ isolated dendrimers adopt different structure types (globular, extended...), but with little apparent correlation between sequence and structure. Sequence matters somewhat more for the dynamics of the dendrimers; however, the choice of residues seems more important close to the galactose substituents than close to the dendrimer stem, where different sequences can yield indis- 
tinguishable dynamics. As a consequence, the sequences providing comparable distance distributions of galactose residues and LecA active sites only show a rather weak specificity for apolar aminoacids toward the end of the dendrimer arms. This is reminiscent of experimental observations about the recognition of LecB by comparable peptide dendrimers, in which the degree of multivalency of the dendrimers was found to have more effect than the dendrimer sequence: despite an apparent preference for positively charged aminoacids, hydrophobic and anionic sequences with comparable binding avidities were identified. ${ }^{48}$ Considering these points, it is all the more interesting that the multivalent binding of dendrimer ligand functions to LecA active sites in a mixed dendrimer/lectin solution shows a marked sequence effect on the two cases studied here. In any case, the traditional structure-activity relationship paradigm which has been a staple of in silico drug design for many years ${ }^{54}$ should be replaced, for the dendrimers under study but also for other conformationnally flexible and/or multivalent drugs, by the concept of dynamics-activity realtionship, in which molecular dynamics methods will naturally play a prominent role.

Multivalent binding comes in several flavors. ${ }^{10}$ The most straightforward, chelation, is achieved by simultaneously binding two or more binding sites on the same receptor with as many groups on the same ligand. A LecA tetramer features 4 binding sites, roughly arranged at the vertices of a rectangle. The small side of this rectangle measures $26 \AA$, which is close to the upper limit of distances observed between dendrimer galactose moieties in the simulations performed herein (see Supporting information, Figure S2). Consequently, while this mode of multivalency cannot be ruled out in these particular systems, it is entropically disfavored. Although the sequence has an impact on the extension of the galactose distance distribution to larger values (for instance, arginine residues at the end of the arms promote extended conformations), this impact is too limited to provide room for improvement without modifying the dendrimer topology. This conclusion is in line with experimental findings on the binding of LecA and LecB by similar multivalent scaffolds; ${ }^{15}$ however, it has very recently been experimentally demonstrated that 4-generation peptidic dendrimer scaffolds (featuring 
4 levels of branching and 16 sugar moieties), which could in theory span larger extents of space than the 3-generation dendrimers discussed here, showed in fact reduced multivalent potency. ${ }^{55}$ Although the sterical clashes between lectin tetramers around each dendrimer, which the authors hold responsible for this phenomenon, is not due to chelation multivalency only, there appears to be little point in seeking to lengthen the dendrimer 'arms'.

Another multivalent mode, dubbed clustering, involves several receptors coming in close vicinity and being subsequently bound by a single, multivalent ligand. This is likely what happens with the worst dendrimer candidate: it is able to modify the dynamics of the LecA tetramers, bringing them together to form long-lasting structural aggregates which it could then, in theory, multivalently bind. From the resuts presented herein, this approach seems only moderately effective: despite promising contact probabilities, the number of simulaneously contacted LecA tetramers appears limited to two at relevant distances. However, it has to be reminded that the so-called 'worst' dendrimer has not been optimized to achieve clustering multivalency: it was chosen within the pool of possible dendrimer sequences to maximize the difference between the distribution of galactose distances and the distribution of active sites in a solution of LecA tetramers. The modifications that the dendrimer triggers in the latter were not expected; consequently, other dendrimer sequences might have an even more pronounced effect, which could result in a more effective clustering-type multivalency. The effect of the dendrimer on the dynamics of LecA is in line with the experimental finding that even a moderate dendrimer stoechiometry can significantly impact the global organization of lectins, while higher concentrations have an even more pronounced effect. ${ }^{52}$ The dendrimer acts as a tensioactive molecule, decreasing lectin-lectin interactions and creating new structures by intercalating between lectins. It has recently been suggested that, for the inhibition of $P$. aeruginosa biofilms, the capacity of a dendrimer to aggregate lectins might be more important than its capacity to bind individual lectins strongly. ${ }^{51}$ Considering the size of the $\left(\mathrm{LecA}_{4}\right)_{5}$-(dendrimer $)_{5}$ system and the timescales involved, optimizing the dendrimer sequence to this effect using MD simulations will be very costly, even at the coarse-grain 
level.

The last instance of multivalent binding mechanisms is called statistical rebinding: a high concentration and/or high mobility of receptors and ligands allows multiple, shortlived interactions between both species, in which contacts that break are quickly replaced by other equivalent ones. From the results presented above, the best dendrimer candidate optimizes this multivalent mechanism, by mimicking the distribution of LecA active site distances without affecting it. Dendrimers with high multivalency are particularly suited to this purpose; indeed, a sharp drop in effeciency was experimentally observed when going from octavalent to tetravalent or trivalent dendrimers of the same sequence in the case of LecB binding (with the latter not being significantly more active than fucose itself). ${ }^{48}$ The importance of multivalency was similarly demonstrated on LecA. ${ }^{56}$ Moreover, the statistical rebinding mechanism explains the broad distributions of the number of effective partners in LecA/dendrimer complexes that have been observed both experimentally ${ }^{57}$ and in the simulations presented here. As for clustering multivalency, there is no guarantee that the 'best' dendrimer suggested in this work has the optimal sequence to maximize statistical rebinding; however, it does seem a good tradeoff between high LecA mobility and relevant interaction lifetimes.

In fact, much like the 'worst' dendrimer candidate profoundly modifies the dynamics of a solution of LecA tetramers, transient interactions between both species (at the active sites or otherwise) have a good chance of affecting the distribution of dendrimer galactose distances. As such, searching for the optimal sequence on the basis of the distance distributions of the isolated dendrimers will only reveal part of the global picture. Since the 'best' dendrimer candidate was shown not to affect the dynamics of the lectin active sites, a model akin to the kinetic model of protein-protein association can be considered: ${ }^{58}$ the formation of the precomplex (a structure in which ligand and receptor are aligned and primed for binding, but still do not form strong interactions) is the obligatory prerequisite to the binding process, while noncognate contacts from random encounters between partners quickly dissociate. In 
this model, the dynamic features of the precomplex can reasonably be approximated from the dynamics of the isolated partners. This has been verified for the 'best' dendrimer candidate, in which $92 \%$ of noncognate contacts (defined as distances smaller than $10 \AA$ between any atoms of the partners belonging neither to the galactose residues nor to the lectin active site aminoacids) last less than 200 ps, versus more than 5 ns for $73 \%$ of cognate contacts. Additionally, this model is even more likely to hold true at lower dendrimer concentrations; it might not, however, apply to dendrimers which have a strong impact on lectin dynamics. So while the dynamics of the isolated dendrimers definitely are a relevant indicator of multivalency (as the prediction of leafward prolines by the model, in agreement with experiments, indicates), it is only one part of a more complex equation. Unfortunately, the simulation of lectin-dendrimer mixtures over relevant timescales is much too costly to allow the screening of a large number of possible dendrimer sequences; in fact, the nature, occurence and impact of dendrimer-LecA interactions are likely to depend on the absolute and relative concentrations of both species which, as previously mentioned, have to be overestimated to bring down computational costs to acceptable levels.

Lectin-based design, which focuses on the structural and dynamical properties of lectins as the basis for the conception of potent ligands, has been the driving force behind the vast majority of studies in the field (including the present one). ${ }^{12}$ However, the profound impact of the dendrimer sequence on the dynamics of LecA tetramers, revealed in this work, calls for a re-evaluation of this traditional assumption, toward a more 'ligand-centric' approach in which the ligands and lectins, rather than the lectins alone, are considered simultaneously from the start. This approach involves very large systems over extensive timeframes, pushing the boundaries of coarse-graining techniques and providing incentive for the development of robust methods combining a simplified representation of the systems with enhanced conformational sampling techniques. 


\section{Associated Content}

Supporting Information. Validation of the branching lysine coase-grain model against allatom data; distributions of sugar distances for cluster exemplars; histogram of KS distances between sugar distance and LecA site distance distributions for all dendrimer sequences; convergence assessment of LecA site distance distributions; validation of the inclusion of proline inside the group of hydrophobic aminoacids.

\section{Author Information}

Funding. The author is employed by Centre National de la Recherche Scientifique.

\section{Acknowledgements}

I thank Mohammed Benazza for fruitful discussions leading to this work. The calculations

presented herein were performed using HPC resources from the MeCS computing platform of Université de Picardie - Jules Verne, Amiens, France.

\section{References}

(1) Gabius, H.-J. J.; André, S.; Jiménez-Barbero, J.; Romero, A.; Solís, D. From Lectin Structure to Functional Glycomics: Principles of the Sugar Code. Trends Biochem. Sci. 2011, 36, 298-313.

(2) Pinho, S. S.; Reis, C. A. Glycosylation in Cancer: Mechanisms and Clinical Implications. Nat. Rev. Cancer 2015, 15, 540-555.

(3) Paleček, E.; Tkáč, J.; Barto` sík, M.; Bertók, T. s.; Ostatná, V.; Paleček, J. Electrochemistry of Nonconjugated Proteins and Glycoproteins. Toward Sensors for Biomedicine and Glycomics. Chem. Rev. 2015, 115, 2045-2108. 
(4) André, S.; Kaltner, H.; Manning, J. C.; Murphy, P. V.; Gabius, H.-J. J. Lectins: Getting Familiar with Translators of the Sugar Code. Molecules 2015, 20, 1788-1823.

(5) Buckeridge, M. S.; de Souza, A. P. Breaking the Glycomic Code of Cell Wall Polysaccharides May Improve Second-Generation Bioenergy Production from Biomass. Bioenergy Res. 2014, 7, 1065-1073.

(6) Mason, C. P.; Tarr, A. W. Human Lectins and their Roles in Viral Infections. Molecules 2015, 20, 2229-2271.

(7) Jo, E.-K. K. Mycobacterial Interaction with Innate Receptors: TLRs, C-Type Lectins, and NLRs. Curr. Opin. Infect. Dis. 2008, 21, 279-286.

(8) Jiménez Blanco, J. L.; Ortiz Mellet, C.; García Fernández, J. M. Multivalency in Heterogeneous Glycoenvironments: Hetero-Glycoclusters, -Glycopolymers and Glycoassemblies. Chem. Soc. Rev. 2013, 42, 4518-4531.

(9) Lundquist, J. J.; Toone, E. J. The Cluster Glycoside Effect. Chem. Rev. 2002, 102, $555-578$.

(10) Levine, P. M.; Carberry, T. P.; Holub, J. M.; Kirshenbaum, K. Crafting Precise Multivalent Architectures. MedChemComm 2013, 4, 493-509.

(11) Compain, P.; Bodlenner, A. The Multivalent Effect in Glycosidase Inhibition: a New, Rapidly Emerging Topic in Glycoscience. ChemBioChem 2014, 15, 1239-1251.

(12) Cecioni, S.; Imberty, A.; Vidal, S. Glycomimetics Versus Multivalent Glycoconjugates for the Design of High Affinity Lectin Ligands. Chem. Rev. 2015, 115, 525-561.

(13) Kiessling, L. L.; Gestwicki, J. E.; Strong, L. E. Synthetic Multivalent Ligands in the Exploration of Cell-Surface Interactions. Curr. Opin. Chem. Biol. 2000, 4, 696-703.

(14) Wittmann, V.; Pieters, R. J. Bridging Lectin Binding Sites by Multivalent Carbohydrates. Chem. Soc. Rev. 2013, 42, 4492-4503. 
(15) Bernardi, A.; Jiménez-Barbero, J.; Casnati, A.; De Castro, C.; Darbre, T.; Fieschi, F.; Finne, J.; Funken, H.; Jaeger, K.-E. E.; Lahmann, M.; Lindhorst, T. K.; Marradi, M.; Messner, P.; Molinaro, A.; Murphy, P. V.; Nativi, C.; Oscarson, S.; Penadés, S.; Peri, F.; Pieters, R. J.; Renaudet, O.; Reymond, J.-L. L.; Richichi, B.; Rojo, J.; Sansone, F.; Schäffer, C.; Turnbull, W. B.; Velasco-Torrijos, T.; Vidal, S.; Vincent, S.; Wennekes, T.; Zuilhof, H.; Imberty, A. Multivalent Glycoconjugates as Anti-Pathogenic Agents. Chem. Soc. Rev. 2013, 42, 4709-4727.

(16) Giuliani, A.; Rinaldi, A. C. Beyond Natural Antimicrobial Peptides: Multimeric Peptides and Other Peptidomimetic Approaches. Cell Mol. Life Sci. 2011, 68, 2255-2266.

(17) Sommer, P.; Fluxa, V. S.; Darbre, T.; Reymond, J.-L. L. Proteolysis of Peptide Dendrimers. ChemBioChem 2009, 10, 1527-1536.

(18) Stach, M.; Siriwardena, T. N.; Köhler, T.; van Delden, C.; Darbre, T.; Reymond, J.L. L. Combining Topology and Sequence Design for the Discovery of Potent Antimicrobial Peptide Dendrimers Against Multidrug-Resistant Pseudomonas Aeruginosa. Angew. Chem. Int. Ed. Engl. 2014, 53, 12827-12831.

(19) Ravi, H. K.; Stach, M.; Soares, T. A.; Darbre, T.; Reymond, J.-L. L.; Cascella, M. Electrostatics and Flexibility Drive Membrane Recognition and Early Penetration by the Antimicrobial Peptide Dendrimer Bh1. Chem. Commun. (Camb.) 2013, 49, 88218823.

(20) Novoa, A.; Eierhoff, T.; Topin, J.; Varrot, A.; Barluenga, S.; Imberty, A.; Römer, W.; Winssinger, N. A LecA Ligand Identified from a Galactoside-Conjugate Array Inhibits Host Cell Invasion by Pseudomonas Aeruginosa. Angew. Chem. Int. Ed. Engl. 2014, 53, 8885-8889.

(21) Kadam, R. U.; Bergmann, M.; Hurley, M.; Garg, D.; Cacciarini, M.; Swiderska, M. A.; Nativi, C.; Sattler, M.; Smyth, A. R.; Williams, P.; Cámara, M.; Stocker, A.; Darbre, T.; 
Reymond, J.-L. L. A Glycopeptide Dendrimer Inhibitor of the Galactose-Specific Lectin LecA and of Pseudomonas Aeruginosa Biofilms. Angew. Chem. Int. Ed. Engl. 2011, 50, 10631-10635.

(22) Kilpatrick, D. C. Animal Lectins: a Historical Introduction and Overview. Biochim. Biophys. Acta 2002, 1572, 187-197.

(23) Mitchell, E.; Houles, C.; Sudakevitz, D.; Wimmerova, M.; Gautier, C.; Pérez, S.; Wu, A. M.; Gilboa-Garber, N.; Imberty, A. Structural Basis for OligosaccharideMediated Adhesion of Pseudomonas Aeruginosa in the Lungs of Cystic Fibrosis Patients. Nat. Struct. Biol. 2002, 9, 918-921.

(24) Filipe, L. C. S.; Machuqueiro, M.; Baptista, A. M. Unfolding the Conformational Behavior of Peptide Dendrimers: Insights from Molecular Dynamics Simulations. J. Am. Chem. Soc. 2011, 133, 5042-5052.

(25) Filipe, L. C.; Machuqueiro, M.; Darbre, T.; Baptista, A. n. M. Unraveling the Conformational Determinants of Peptide Dendrimers Using Molecular Dynamics Simulations. Macromolecules 2013, 46, 9427-9436.

(26) Johansson, E. M.; Kolomiets, E.; Rosenau, F.; Jaeger, K.-E. . E.; Darbre, T.; Reymond, J.-L. . L. Combinatorial Variation of Branching Length and Multivalency in a Large (390625 Member) Glycopeptide Dendrimer Library: Ligands for Fucose-Specific Lectins. New J. Chem. 2007, 31, 1291-1299.

(27) Fritz, D.; Koschke, K.; Harmandaris, V. A.; van der Vegt, N. F. A.; Kremer, K. Multiscale Modeling of Soft Matter: Scaling of Dynamics. Phys. Chem. Chem. Phys. 2011, 13, 10412-10420.

(28) Monticelli, L.; Kandasamy, S. K.; Periole, X.; Larson, R. G.; Tieleman, D. P.; Marrink, S.-J. . J. The MARTINI Coarse-Grained Force Field: Extension to Proteins. J. Chem. Theory Comput. 2008, 4, 819-834. 
(29) Marrink, S. J.; Risselada, H. J.; Yefimov, S.; Tieleman, D. P.; de Vries, A. H. The MARTINI Force Field: Coarse Grained Model for Biomolecular Simulations. J. Phys. Chem. B 2007, 111, 7812-7824.

(30) Zhao, J.; Zhao, C.; Liang, G.; Zhang, M.; Zheng, J. Engineering Antimicrobial Peptides with Improved Antimicrobial and Hemolytic Activities. J. Chem. Inf. Model. 2013, 53, $3280-3296$.

(31) Zhao, X.; Yu, H.; Yang, L.; Li, Q.; Huang, X. Simulating the Antimicrobial Mechanism of Human $\beta$-Defensin-3 with Coarse-Grained Molecular Dynamics. J. Biomol. Struct. Dyn. 2015, 33, 2522-2529.

(32) Horn, J. N.; Sengillo, J. D.; Lin, D.; Romo, T. D.; Grossfield, A. Characterization of a Potent Antimicrobial Lipopeptide via Coarse-Grained Molecular Dynamics. Biochim. Biophys. Acta 2012, 1818, 212-218.

(33) Cohen, F. E.; Sternberg, M. J. On the Prediction of Protein Structure: the Significance of the Root-Mean-Square Deviation. J. Mol. Biol. 1980, 138, 321-333.

(34) Yazici, B.; Yolacan, S. A Comparison of Various Tests of Normality. J. Statist. Comput. Simulation 2007, 77, 175-183.

(35) Frey, B. J.; Dueck, D. Clustering by Passing Messages Between Data Points. Science 2007, 315, 972-976.

(36) Schneider, T. D.; Stephens, R. M. Sequence Logos: a New Way to Display Consensus Sequences. Nucleic Acids Res. 1990, 18, 6097-6100.

(37) Pronk, S.; Páll, S.; Schulz, R.; Larsson, P.; Bjelkmar, P.; Apostolov, R.; Shirts, M. R.; Smith, J. C.; Kasson, P. M.; van der Spoel, D.; Hess, B.; Lindahl, E. GROMACS 4.5: a High-Throughput and Highly Parallel Open Source Molecular Simulation Toolkit. Bioinformatics 2013, 29, 845-854. 
(38) Pall, S.; Abraham, M. J.; Kutzner, C.; Hess, B.; Lindahl, E. Solving Software Challenges for Exascale; Springer, 2014; pp 3-27.

(39) López, C. A.; Rzepiela, A. J.; De Vries, A. H.; Dijkhuizen, L.; Hu nenberger, P. H.; Marrink, S. J. Martini Coarse-Grained Force Field: Extension to Carbohydrates. J. Chem. Theory Comput. 2009, 5, 3195-3210.

(40) Fuhrmans, M.; Sanders, B. P.; Marrink, S.-J.; de Vries, A. H. Effects of Bundling on the Properties of the SPC Water Model. Theor. Chem. Acc. 2010, 125, 335-344.

(41) Cioci, G.; Mitchell, E. P.; Gautier, C.; Wimmerová, M.; Sudakevitz, D.; Pérez, S.; Gilboa-Garber, N.; Imberty, A. Structural Basis of Calcium and Galactose Recognition by the Lectin PA-IL of Pseudomonas Aeruginosa. FEBS Lett. 2003, 555, 297-301.

(42) Van Der Walt, S.; Colbert, S. C.; Varoquaux, G. The NumPy Array: a Structure for Efficient Numerical Computation. Comput. Sci. Eng. 2011, 13, 22-30.

(43) Pedregosa, F.; Varoquaux, G.; Gramfort, A.; Michel, V.; Thirion, B.; Grisel, O.; Blondel, M.; Prettenhofer, P.; Weiss, R.; Dubourg, V. Scikit-learn: Machine Learning in Python. J. Mach. Learn. Res. 2011, 12, 2825-2830.

(44) Humphrey, W.; Dalke, A.; Schulten, K. VMD: Visual Molecular Dynamics. J. Mol. Graphics Modell. 1996, 14, 33-38.

(45) Crooks, G. E.; Hon, G.; Chandonia, J.-M. M.; Brenner, S. E. WebLogo: a Sequence Logo Generator. Genome Res. 2004, 14, 1188-1190.

(46) Huerta-Cepas, J.; Dopazo, J.; Gabaldón, T. ETE: a Python Environment for Tree Exploration. BMC Bioinformatics 2010, 11, 24-30.

(47) Hunter, J. D. Matplotlib: a 2D Graphics Environment. Comp. Sci. Eng. 2007, 9, 90-95.

(48) Kolomiets, E.; Swiderska, M. A.; Kadam, R. U.; Johansson, E. M. V.; Jaeger, K.-E. E.; Darbre, T.; Reymond, J.-L. L. Glycopeptide Dendrimers with High Affinity for the 
Fucose-Binding Lectin LecB from Pseudomonas Aeruginosa. ChemMedChem 2009, 4, $562-569$.

(49) Zamyatnin, A. A. Protein Volume in Solution. Prog. Biophys. Mol. Biol. 1972, 24, $107-123$.

(50) Kyte, J.; Doolittle, R. F. A Simple Method for Displaying the Hydropathic Character of a Protein. J. Mol. Biol. 1982, 157, 105-132.

(51) Visini, R.; Jin, X.; Bergmann, M.; Michaud, G.; Pertici, F.; Fu, O.; Pukin, A.; Branson, T. R.; Thies-Weesie, D. M. E.; Kemmink, J.; Gillon, E.; Imberty, A.; Stocker, A.; Darbre, T.; Pieters, R. J.; Reymond, J.-L. L. Structural Insight into Multivalent Galactoside Binding to Pseudomonas Aeruginosa Lectin LecA. ACS Chem. Biol. 2015, 10, $2455-2462$.

(52) Sicard, D.; Chevolot, Y.; Souteyrand, E.; Imberty, A.; Vidal, S.; Phaner-Goutorbe, M. Molecular Arrangement Between Multivalent Glycocluster and Pseudomonas Aeruginosa LecA (PA-IL) by Atomic Force Microscopy: Influence of the Glycocluster Concentration. J. Mol. Recogn. 2013, 26, 694-699.

(53) Stark, A. C.; Andrews, C. T.; Elcock, A. H. Toward Optimized Potential Functions for Protein-Protein Interactions in Aqueous Solutions: Osmotic Second Virial Coefficient Calculations Using the MARTINI Coarse-Grained Force Field. J. Chem. Theory Comput. 2013, 9, 4176-4185.

(54) Bohacek, R. S.; McMartin, C.; Guida, W. C. The Art and Practice of Structure-Based Drug Design: a Molecular Modeling Perspective. Med. Res. Rev. 1996, 16, 3-50.

(55) Bergmann, M.; Michaud, G.; Visini, R.; Jin, X.; Gillon, E.; Stocker, A.; Imberty, A.; Darbre, T.; Reymond, J.-L. L. Multivalency Effects on Pseudomonas Aeruginosa Biofilm Inhibition and Dispersal by Glycopeptide Dendrimers Targeting Lectin LecA. Org. Biomol. Chem. 2016, 14, 138-148. 
(56) Gestwicki, J. E.; Strong, L. E.; Kiessling, L. L. Tuning Chemotactic Responses with Synthetic Multivalent Ligands. Chem. Biol. 2000, 7, 583-591.

(57) Mullen, D. G.; Fang, M.; Desai, A.; Baker, J. R.; Orr, B. G.; Banaszak Holl, M. M. A Quantitative Assessment of Nanoparticle-Ligand Distributions: Implications for Targeted Drug and Imaging Delivery in Dendrimer Conjugates. ACS Nano 2010, 4, 657670.

(58) Schreiber, G.; Haran, G.; Zhou, H.-X. X. Fundamental Aspects of Protein-Protein Association Kinetics. Chem. Rev. 2009, 109, 839-860.

Figure 1: 2D sketch of the multivalent glycoconjugated peptide dendrimers under study. The lysine residues which implement branching via side-chain isopeptide bonds are framed.

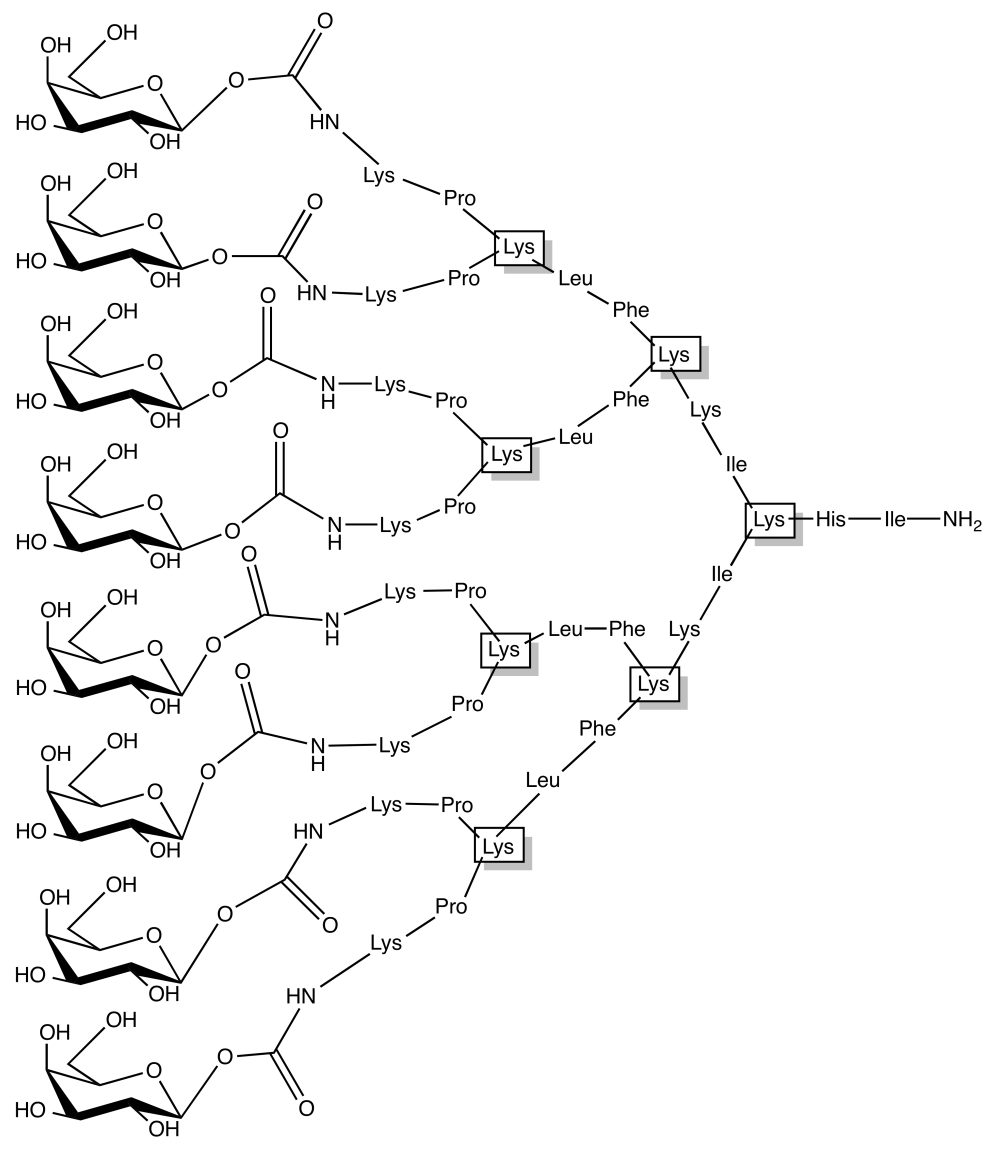


Figure 2: Properties of the clusters inferred from the RMSD matrix between the average structures of the dendrimers. Each row (except the last) represents a cluster, characterized by (i) its member count (white numbers on left panel), (ii) statistics over mutation positions (left panel, pie chart using the position of the mutated aminoacid closest to the dendrimer trunk and the color code given on the last line, left panel), (iii) hydropathy indices (center panel, red bars, sum of values over the two mutated aminoacids) and (iv) volume (right panel, green bars, sum of values over the two mutated aminoacids). The hydropathy and volume bar plots on the last line correspond to the entire population of 175 dendrimers. Hydropathy values are binned from -9.0 (left bar, light red) to 3.8 (right bar, dark red) in steps of $~ 1.4$; volumes are binned from $106.4 \mathrm{~mL} \mathrm{~mol}^{-1}$ (left bar, light green) to $208.2 \mathrm{~mL}$ $\mathrm{mol}^{-1}$ (right bar, dark green) in steps of $\sim 11.4 \mathrm{~mL} \mathrm{~mol}^{-1}$.
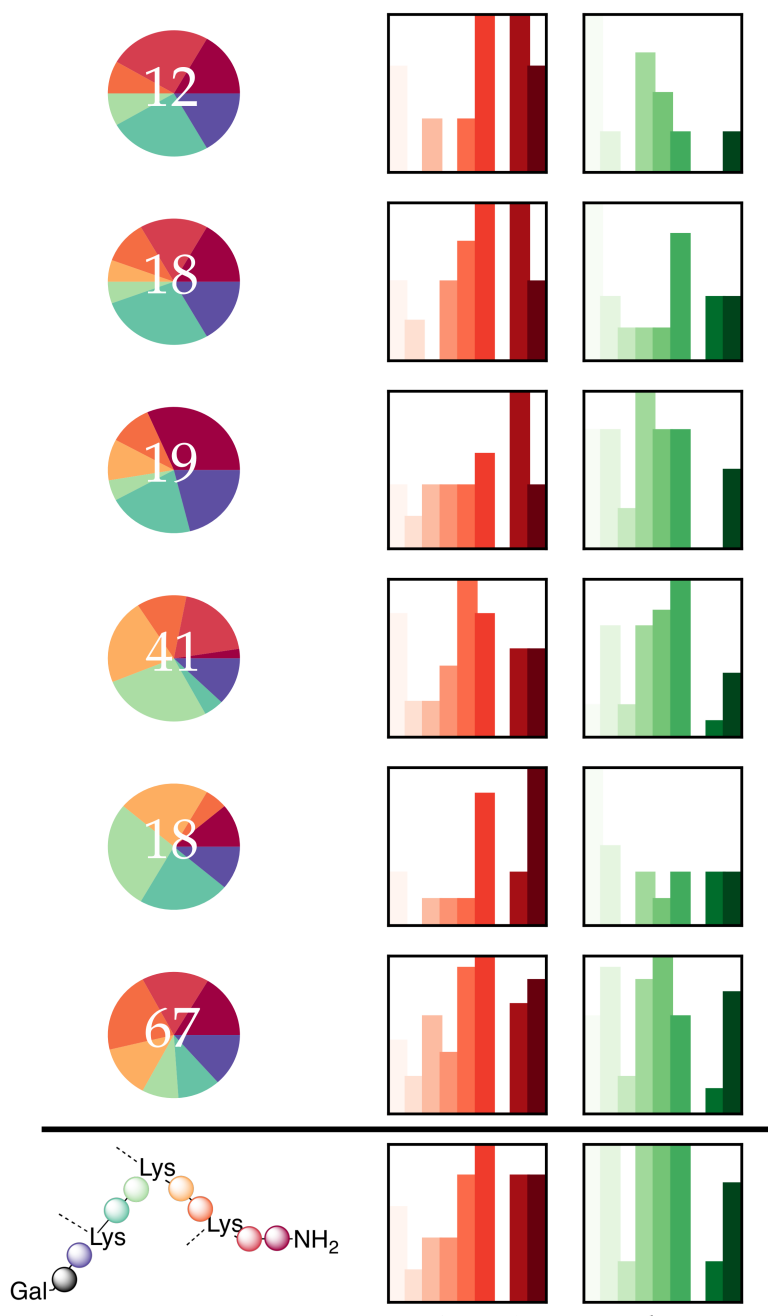

Hydropathy

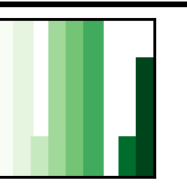

index

Volume 
Figure 3: Top panel: superposition of the representative structures of the clusters (as backbone bead surfaces, with one color per cluster). The structures are aligned along their inertial axes (first axis $\mathrm{I}_{1}$ : horizontal; second axis $\mathrm{I}_{2}$ : vertical; third axis $\mathrm{I}_{3}$ : perpendicular to the figure plane). Bottom panels: moments along each inertial axis for each cluster representative (colors match the top panel structures).
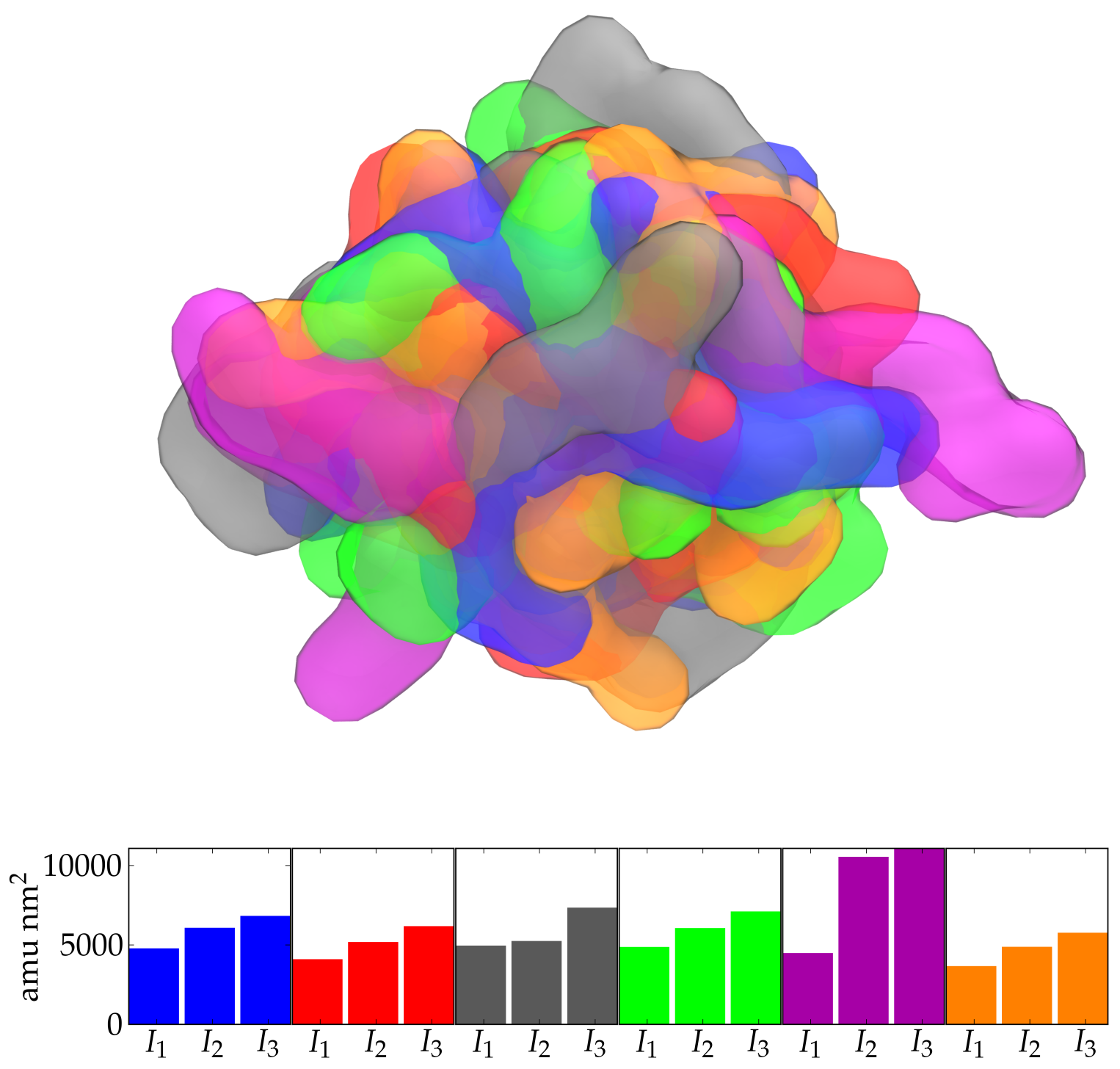
Figure 4: Hierarchical clutering of dendrimer sequences with respect to the distributions of inter-sugar distances over time. The filiation of clusters is represented as a tree, from center (parents) toward periphery (children). Each cluster is described by its member count and statistics over mutation positions (pie chart, using the position of the mutated aminoacid closest to the dendrimer trunk), hydropathy index (red bars, sum of values over the two mutated aminoacids) and volume (green bars, sum of values over the two mutated aminoacids).

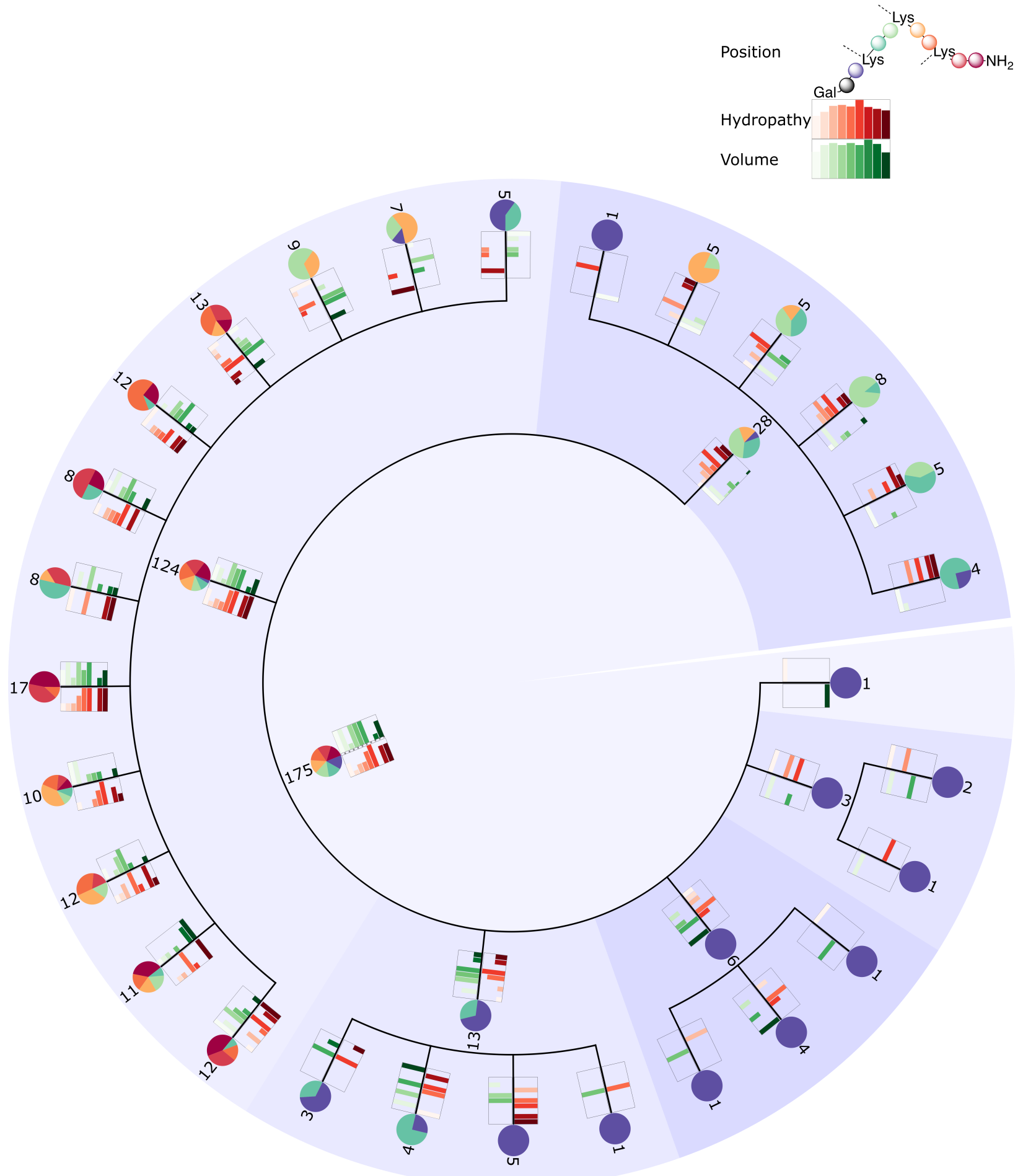


Figure 5: Left panel: mean and standard deviation of KS distances between sugar distance distributions for (i) pairs of dendrimers bearing a specific residue at a specific location (solid black line/circles) and (ii) a dendrimer bearing a specific residue at a specific location and any other dendrimer (dotted red line/squares). Right panel: Mean and standard deviation of KS distances between sugar distance distributions for pairs of dendrimers mutated at a given position which have been simultaneously mutated (i) on the left (trunkward) side (red), (ii) on the right (leafward) side (blue), (iii) on either side (black).
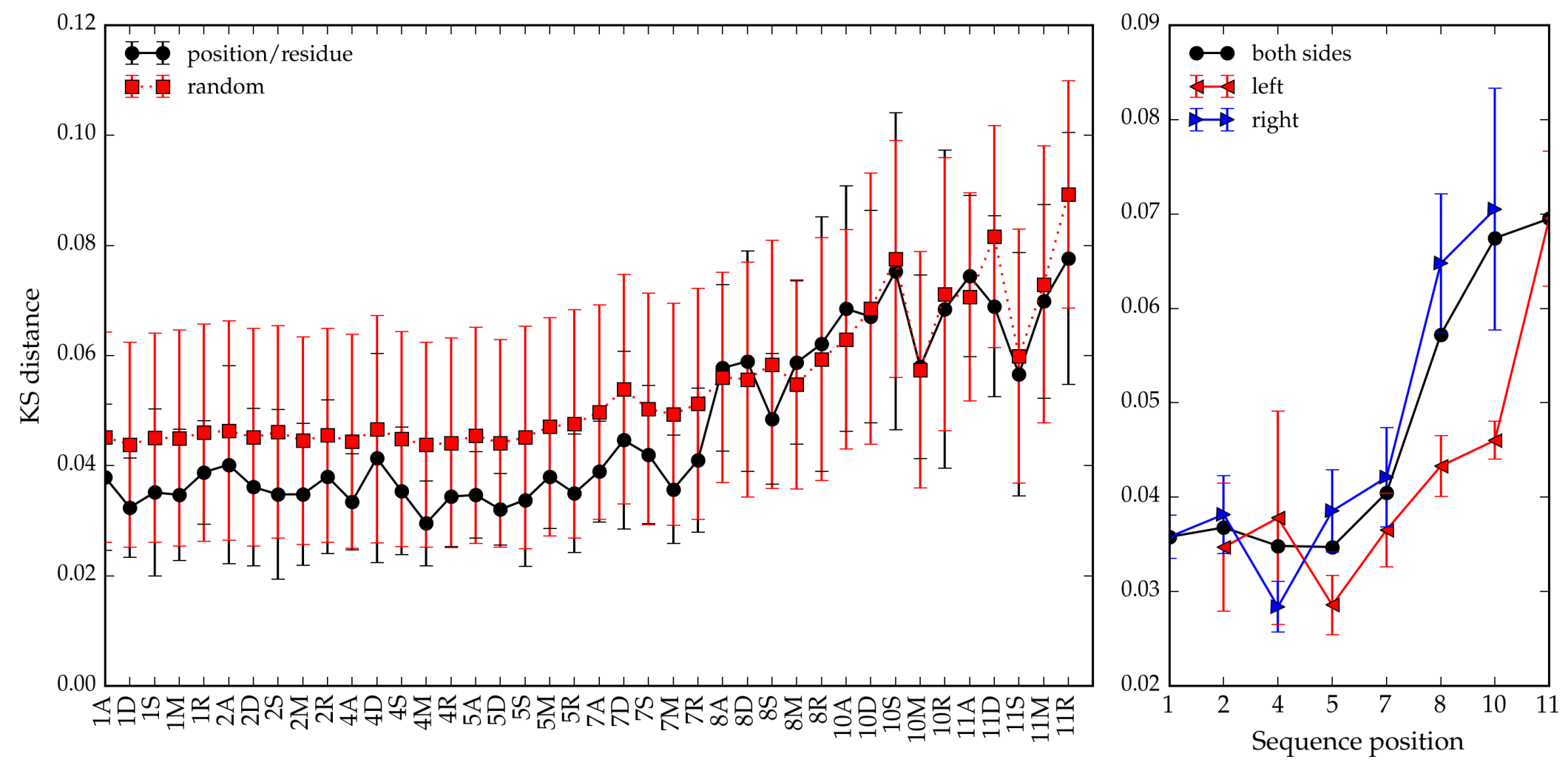
Figure 6: Putative dendrimer sequence preferences for the multivalent binding of LecA. The height of each letter is proportional to its statistical relevance in the set of sequences for which the overlap of ligand and receptor distance distributions is optimal. The sequence is ordered from trunk (left) to leaves (right).

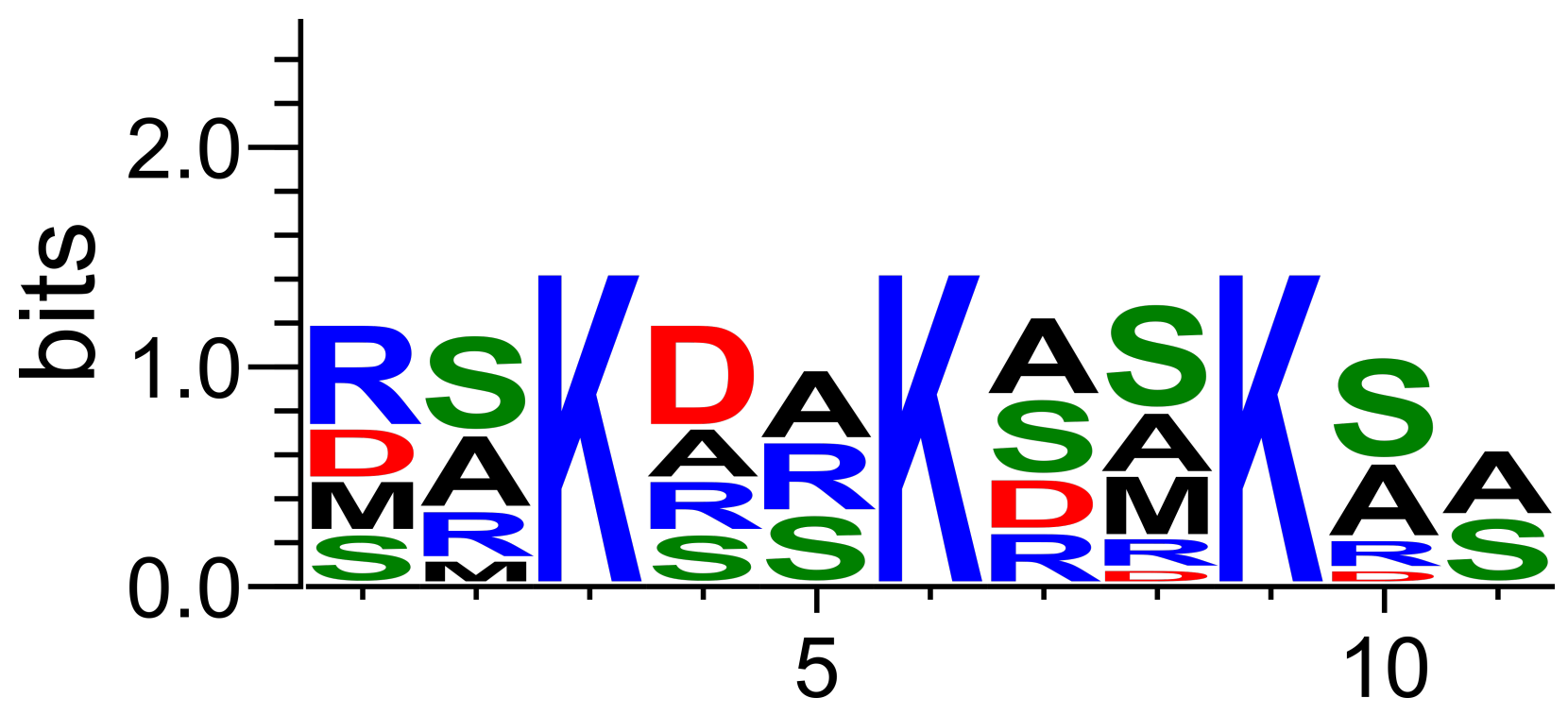


Figure 7: Distribution of distances between active sites on distinct LecA tetramers: in the absence of dendrimers (top); in the presence of the best (middle) and worst (bottom) dendrimer candidates.

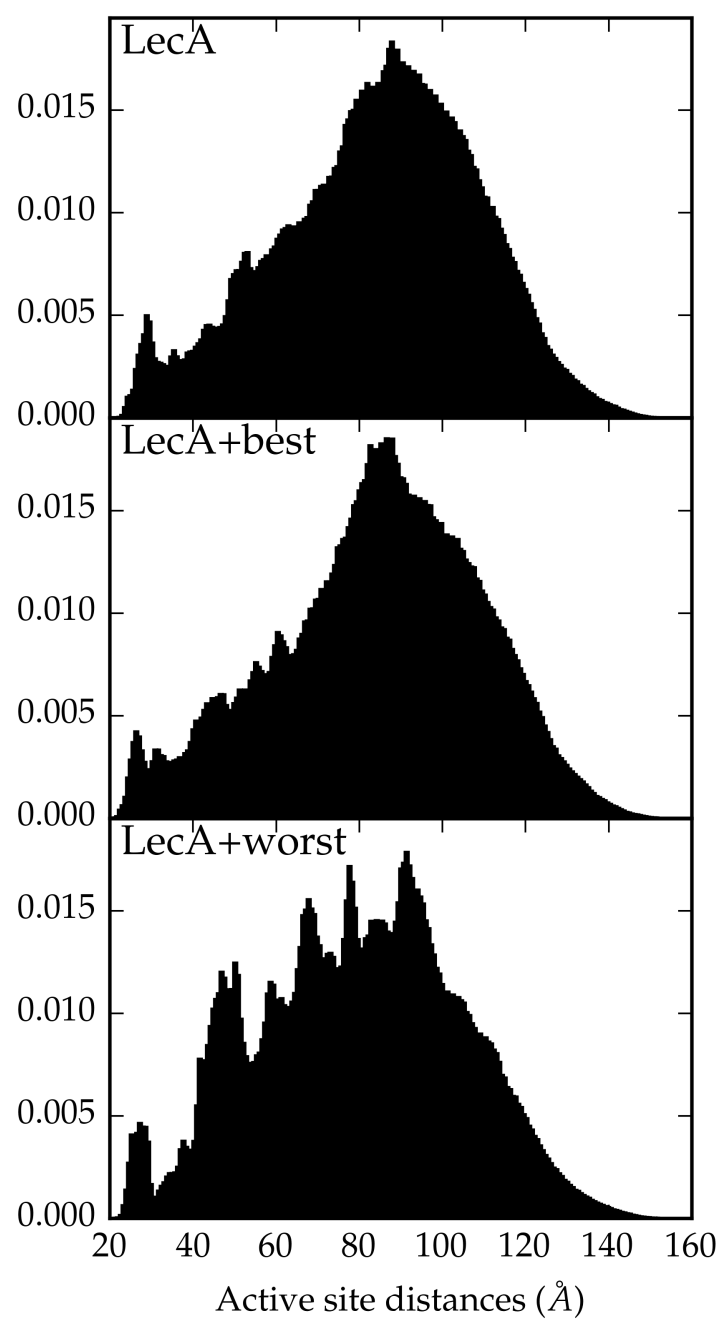


Figure 8: Coarse-grained structural representation of the 'best' galactopeptide dendrimer (white sticks: peptidic arms; blue triangles: galactose) multivalently bound to three LecA tetramers (yellow/red surface), extracted from the corresponding molecular dynamics simulation. LecA active sites can be spotted by their $\mathrm{Ca}^{2+}$ ions (green spheres).

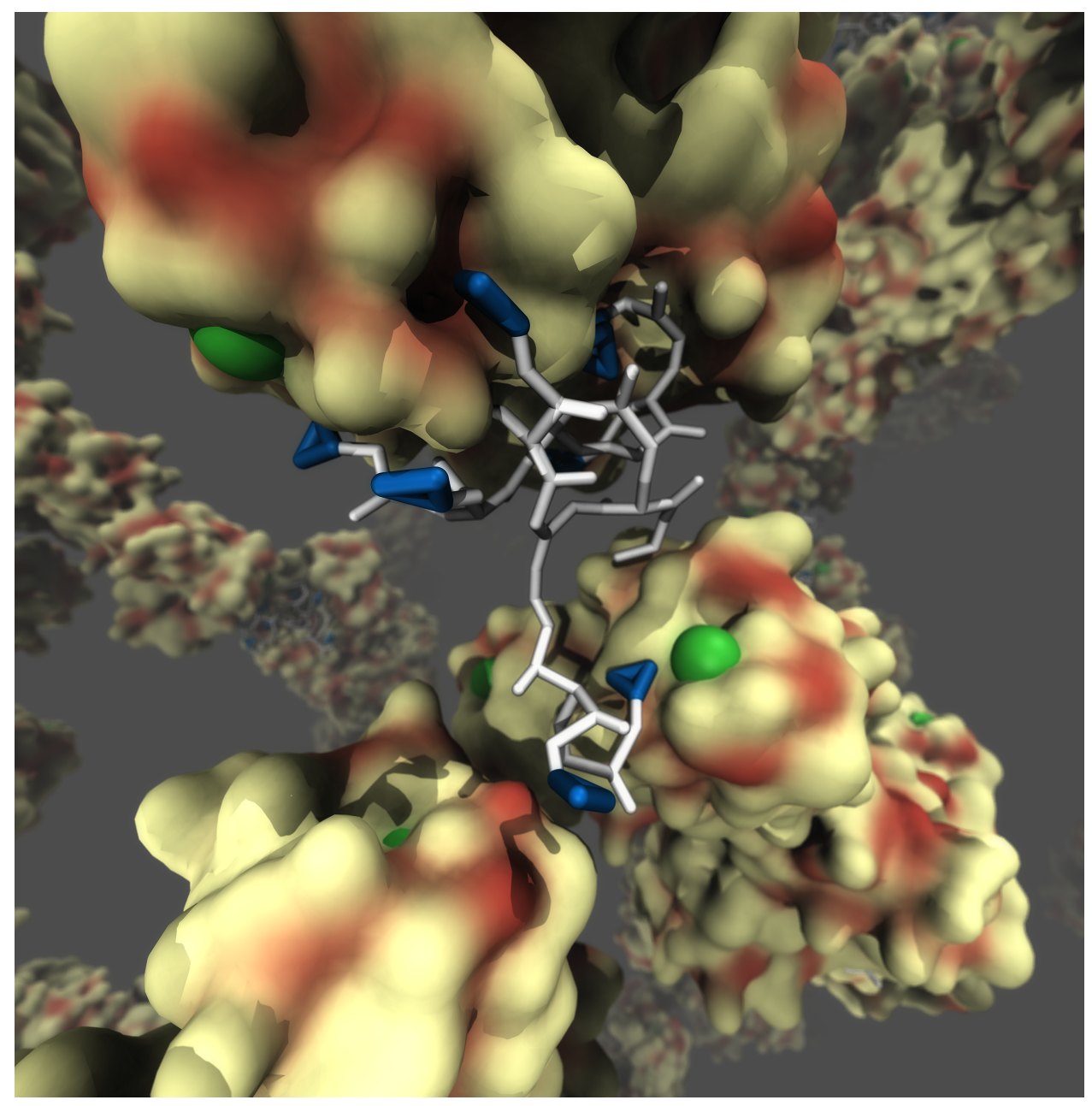


Figure 9: Probability of simultaneously finding one to five LecA tetramers active sites within a given distance of a dendrimer galactose, for the best (top) and worst (bottom) dendrimer candidates in solution with LecA.

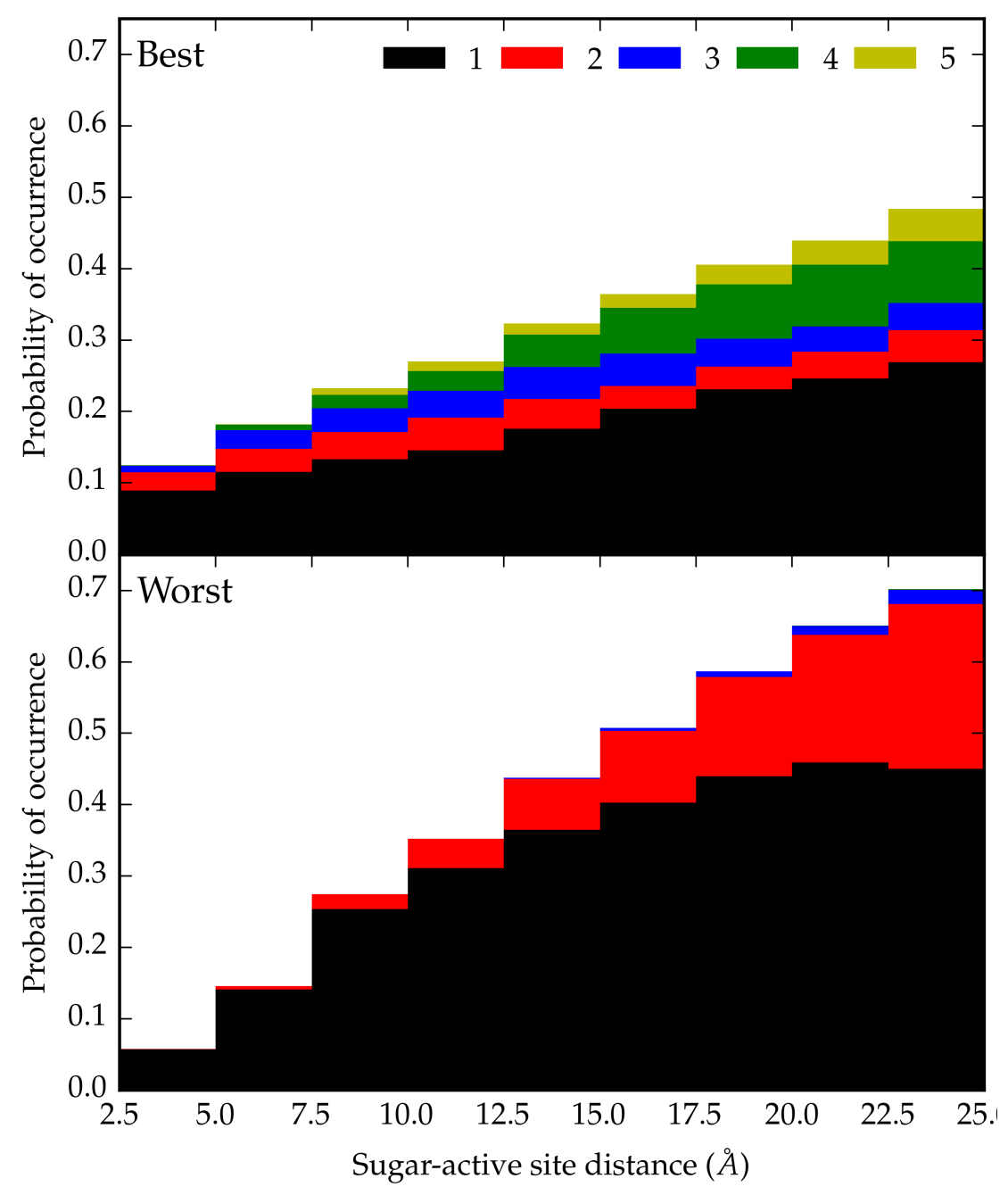




\section{Graphical TOC Entry}

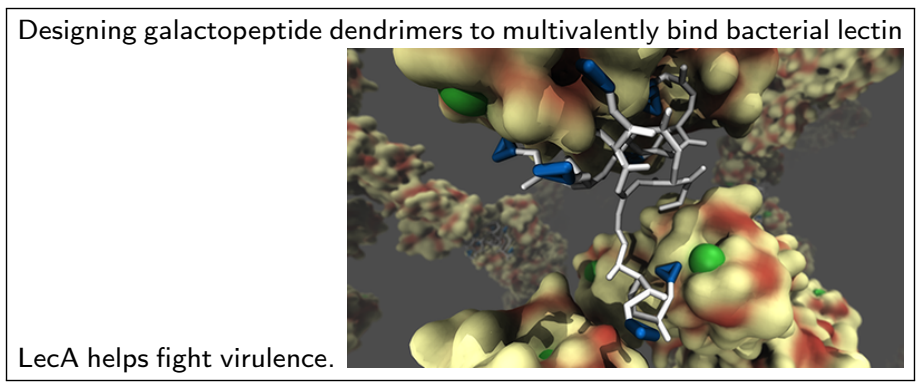

